\title{
Live Intravital Intestine with Blood Flow Visualization in Neonatal Mice Using Two-photon Laser
} Scanning Microscopy

\author{
Yuhki Koike ${ }^{1,2}$, Bo Li $i^{1}$, Yong Chen ${ }^{1}$, Niloofar Ganji ${ }^{1}$, Mashriq Alganabi ${ }^{1}$, Hiromu Miyake ${ }^{1}$, Carol Lee ${ }^{1}$, \\ Alison Hock ${ }^{1}$, Richard Wu ${ }^{3}$, Keiichi Uchida², Mikihiro Inoue ${ }^{2}$, Paul Delgado-Olguin ${ }^{4}, 5,6$, Agostino
}

Pierro ${ }^{1, *}$

${ }^{1}$ Division of General and Thoracic Surgery, Physiology and Experimental Medicine Program, The Hospital for Sick Children, Toronto, ON, Canada; ${ }^{2}$ Department of Gastrointestinal and Pediatric Surgery, Mie University Graduate School of Medicine, Tsu, Mie, Japan; ${ }^{3}$ Division of Gastroenterology, Hepatology and Nutrition, The Hospital for Sick Children, Toronto, ON, Canada; ${ }^{4}$ Translational Medicine, The Hospital for Sick Children, Toronto, Ontario, Canada; ${ }^{5}$ Department of Medical Biophysics, University of Toronto, Toronto, Ontario, Canada; ${ }^{6}$ Heart \& Stroke Richard Lewar Centre of Excellence, Toronto, Ontario, Canada

*For correspondence: agostino.pierro@sickkids.ca

[Abstract] This protocol describes a novel technique to investigate the microcirculation dynamics underlying the pathology in the small intestine of neonatal mice using two-photon laser-scanning microscopy (TPLSM). Recent technological advances in multi-photon microscopy allow intravital analysis of different organs such as the liver, brain and intestine. Despite these advances, live visualization and analysis of the small intestine in neonatal rodents remain technically challenging. We herein provide a detailed description of a novel method to capture high resolution and stable images of the small intestine in neonatal mice as early as postnatal day 0 . This imaging technique allows a comprehensive understanding of the development and blood flow dynamics in small intestine microcirculation.

Keywords: Intravital imaging, In vivo imaging, Two-photon laser scanning microscopy (TPLSM), Necrotizing enterocolitis (NEC), Neonatal mouse imaging

\section{[Background]}

\section{Development of in vivo real-time imaging}

Recent technological advances are overcoming the limitations of conventional histological analysis by enabling live imaging on experimental animals. In contrast to conventional histological microscopy techniques, the intravital approach provides insight into previously unknown morphogenetic and functional processes in live tissues. Furthermore, this information can be acquired in real time lapses, whereas alternative techniques are limited to a snapshot in time. The alternative techniques have the added requirement of determining the best timing to acquire images during a specific process in an experimental protocol.

Two-photon laser-scanning microscopy (TPLSM), compared to confocal microscopy, offers in vivo imaging that is superior for deep optical sectioning of living tissue (Pittet and Weissleder, 2011). The 
higher resolution and reduced phototoxicity of this method allow longer time periods of continuous realtime imaging on intact organs. We have standardized in vivo real-time imaging of intra-abdominal organs through TPLSM and used it to study the contribution of abnormal intestinal microcirculation in pathophysiological processes. For example, we have used this technology to study bacterial translocation in dextran sodium sulfate (DSS)-induced colitis (Toiyama et al., 2010), neutrophil extracellular traps (Tanaka et al., 2014a), thrombus formation in laser-induced endothelium injury (Koike et al., 2011), visualization of chemotherapy responses of colorectal liver metastases to the tumor microenvironment (Tanaka et al., 2014b), and the dynamics of circulating free DNA in a model of DSSinduced colitis (Koike et al., 2014). Additionally, previous reports have shown the application of intravital multiphoton microscopy to study pathophysiological processes in other abdominal organs including the liver (Honda et al., 2013; Lu et al., 2014; Liang et al., 2015), pancreas (Coppieters et al., 2010; Martinic and von Herrath, 2008), spleen (Ferrer et al., 2012), and kidneys (Peti-Peterdi et al., 2012; Hackl et al., 2013; Devi et al., 2013; Schiessl and Castrop, 2013).

In recent years TPLSM has become increasingly popular in in vivo research. However, intravital imaging of the small intestine in neonatal mice has been challenging due to their small body size and fragility of the intestinal wall. We have developed a novel application of TPLSM to visualize and study the small intestine of neonatal mice in vivo.

\section{Advantages of the method}

Two of the key advances of TPLSM are deeper tissue penetration and reduced photobleaching. These advances have facilitated the study of dynamic processes in vivo while minimizing injury to the organ or cell under investigation. Obtaining intravital stable images of the gastrointestinal tract has been difficult due to the movements caused by heartbeat, respiration, and intestinal peristalsis. To reduce the effects of these movements, we and others have purposefully designed devices and equipment that improve live image stability of the gastrointestinal tract (Watson et al., 2005; McDole et al., 2012; Ritsma et al., 2013). However, setting up such devices is time consuming, costly, and present technical challenges that require extensive trial and error. Current systems of choice for stabilizing intravital imaging are: a viewing window with a vacuum chamber (Looney et al., 2011), a microstage device (Cao et al., 2012), and our previously designed organ stabilizer (Japanese Patent No. 5268282) (Toiyama et al., 2010). These methods have been used for imaging the small intestine in adult mice, however, none of them have been applied to the imaging of the neonatal small intestine. One reason for this is that these devices are too large and limit the working space for the neonatal small intestine. To limit the movements induced by heartbeat and breathing during imaging, it is important that the organ stabilizer is detached from the neonatal abdominal wall. In addition, fixation maneuvers required when using previously developed organ stabilizing devices can cause injury or affect the neonatal intestinal microcirculation.

Our method allows for direct microvascular blood flow analysis. Stappenbeck TS et al. (2002) reported a method to analyze intestinal microcirculation indirectly from intestinal tissue samples harvested immediately after injecting fluorescein isothiocyanate-labeled dextran into the heart (Yu et al., 2009; Watkins and Besner, 2013; Yazji et al., 2013). However, thisand other similar methods for intravital 
imaging of the adult mouse intestine are not functional in blood flow dynamics. Our method allows for blood flow dynamics analysis, and facilitates investigation of intestinal villi development and establishment of the capillary network complexity (Stappenbeck et al., 2002). This protocol will allow the investigation of pathological processes associated with intestinal blood flow dynamics in vivo, thus promoting translational research.

The method we describe in this protocol is simple, overcomes the limitations of previous systems, and allows for stable live imaging of the neonatal mouse small intestine. This method interferes minimally with the microcirculation and enables high resolution intravital imaging of the small intestine for long periods of time. This protocol allows for unprecedented stability of intravital imaging of the neonatal small intestine.

\section{Applications of the method}

The TPLSM imaging method described here can be easily applied to investigate different physiological processes in the neonatal intestine in multiple mouse models. For example, we are using this method to study blood flow dynamics and inflammatory responses in necrotizing enterocolitis (NEC), intestinal epithelium and micro-vasculature development in short bowel syndrome (SBS), inflammatory and immunological status in inflammatory bowel disease (IBD), and ischemia-reperfusion injury in midgut volvulus. Previous studies investigated neonatal intestinal microcirculation in experimental NEC ex vivo, however, these studies did not consider the effects of blood flow dynamics in capillary-vessels in the villi. Our new method allowed us to measure neonatal intestinal microcirculation from movies of live blood flow and to derive blood flow velocity, vessel diameter and inflammation, and assess irrigation of the serosal and mucosal layers. Moreover, this technique is being used to visualize and quantify live blood flow dynamics during reperfusion and ischemia in experimental midgut volvulus, which will be useful to identify the primary intestinal tissues affected.

Here, we provide a step-by-step methodology to set up the neonatal mouse small intestine using a simple stabilizing device to evaluate the intestinal microcirculation by TPLSM.

\section{Limitation of the approach}

The proximal jejunum close to the ligament of Treitz is difficult to study due to its proximity and attachment to the abdominal wall. The described technique allows for analysis of the small intestine between the anterior superior iliac spine and the xiphoid process transversely, and between the sternum and the posterior abdominal wall longitudinally. Analyzing the intestine outside these marking points (for instance, in portions of the colon) may lead to potential bleeding from the mesentery due to excessive stretching applied on the intestine for appropriate positioning. Additionally, the device is limited to areas of the intestine that are naturally close to the abdominal wall without the need for heavy manipulation to avoid potential intestinal damage. The organ stabilizing device should not be in direct contact with the mouse abdominal wall to avoid image instability caused by movements due to breathing and heartbeat. 


\section{Considerations for intestinal preparation}

The neonatal mouse should be fasted for at least 4 hours before microscopic observation since food residue within the intestinal lumen could affect the imaging results by affecting intestinal blood flow and/or potential for development of ischemia. Some reports have shown that intestinal blood flow varies by gestational age and feeding time (Pezzati et al., 2004; Watkins and Besner, 2013; Thompson et al., 2014; Morgan et al., 2014), suggesting that feeding tolerance should also be considered in the study protocol. Therefore, for analyzing the small intestine microcirculation using this method, a fixed fasting duration and gestational age between for all mice being used should be considered before starting the experiment to allow for appropriate comparison. The ideal fasting time to use will vary depending on the specific parameters to be measured in the neonatal small intestine. For example, 6-hour fasting allows proper imaging of the neonatal ileum in 5-day old pups subjected to experimental NEC, but this may vary if examining a different disease or mice of a different age. The varying effects on blood circulation secondary to feeding should be minimized by maintaining a homogenous feeding schedule and fasting duration across all mice being examined.

\section{Procedure for NEC induction}

NEC is induced by gavage feeding a hyperosmolar formula gavage, exposure to temporal hypoxia and oral administration of lipopolysaccharide (LPS) (Zani et al., 2008). Gavage feeding is given 3 times a day, using a 1.9-Fr silicon catheter (Vygon UK Ltd, Gloucestershire, UK). The hyperosmolar formula is prepared with $15 \mathrm{~g}$ of SMA Gold (SMA Nutrition, Berkshire, UK) in $75 \mathrm{ml}$ of Esbilac canine supplement (Pet-Ag Inc., Hampshire, IL) (Barlow et al., 1974). Pups are exposed to hypoxia before each feed by placing them in a hypoxic chamber containing a gas mixture of $5 \% \mathrm{O}_{2}$ and $95 \% \mathrm{~N}_{2}$ for 10 min, monitored with an $\mathrm{O}_{2}$ gas detector (BW $\mathrm{O}_{2}$ Gas Alert Clip Extreme, Rockall Confined \& Safety, Cardiff, UK). LPS is administered on the 1st and 2nd day after NEC induction; mice are gavaged with $4 \mathrm{mg} / \mathrm{kg} /$ day LPS (lipopolysaccharide from Escherichia coli 0111:B4, Sigma-Aldrich Company Ltd., Dorset, UK) mixed within the formula feed. During the whole experiment, mice are kept in a neonatal incubator to maintain temperature $\left(30^{\circ} \mathrm{C}\right)$ and humidity $(40 \%)$.

\section{Materials and Reagents}

Note: All reagents may be substituted with appropriate alternatives from other manufacturers.

\section{Consumables}

1. Sterile $1.0 \mathrm{ml}$ syringe with 26 Gauge needle or smaller (VWR, catalog number: 309597 )

2. Sterile gauze (VWR, catalog number: CA95041-740)

3. Soft absorbable pad (VWR, catalog number: 95057-862)

4. Rubber grove

5. Kimwipes (VWR, catalog number: 102097-615)

6. Microscope slides (VWR, catalog number: 48311-703, $1.0 \mathrm{~mm}$ thickness)

7. Microscope cover glass (VWR, catalog number: 48393-172, 0.13-0.17 mm thickness) 
8. Falcon tube (15 ml) (VWR, catalog number: CA60819-761)

9. Adhesive tape (VWR, catalog number: 89097-912)

\section{Animals}

Neonatal $R O S A^{m T / m G}$;Tie2-Cre mice of both sexes were used to visualize the hemodynamics of small intestinal microcirculation and leukocyte movement. Table 1 shows a comprehensive list of transgenic mouse lines carrying fluorescent reporters that could be used in this protocol. Alternative transgenic reporter lines expressing fluorescent proteins can also be used in this method.

ICaution: Please note that all animal experiments should be performed following ethical guidelines and regulations of both the animal and imaging facilities. Research should not begin until use live animals is approved by the facility's Animal Care Committee.

Table 1. Fluorescent positive mouse lines

\begin{tabular}{|c|c|c|c|c|}
\hline Name & Strain & Type & Promoter & Specificity \\
\hline GFP mouse & C57BL/6 & $\operatorname{Tg}(C A G-E G F P)$ & $\begin{array}{l}\text { Chicken } \beta \text {-Actin and } \\
\text { cytomegalovirus } \\
\text { enhancer }\end{array}$ & $\begin{array}{l}\text { All tissues except for erythrocytes and hair } \\
\text { appear green under excitation light. }\end{array}$ \\
\hline $\begin{array}{l}\mathrm{mTmG} \\
\text { mouse }\end{array}$ & C57BL/6 & Rosa $^{\mathrm{mTmG}}$ & Chicken $\beta$-Actin/pCA & $\begin{array}{l}\text { These mice possess loxP sites on either } \\
\text { side of a membrane-targeted tdTomato } \\
(\mathrm{mT}) \text { cassette and express strong red } \\
\text { fluorescence in all tissues and cell types } \\
\text { examined. When bred to Cre recombinase } \\
\text { expressing mice, the resulting offspring } \\
\text { have the } \mathrm{mT} \text { cassette deleted in } \\
\text { the Cre expressing tissue(s), allowing } \\
\text { expression of the membrane-targeted } \\
\text { EGFP (mG) cassette located just } \\
\text { downstream. }\end{array}$ \\
\hline Tie2 Cre & $\begin{array}{l}\text { (C57BL/6 x } \\
\text { SJL)F1 }\end{array}$ & Tek-cre & $\begin{array}{l}\text { Tek, endothelial- } \\
\text { specific receptor } \\
\text { tyrosine kinase }\end{array}$ & $\begin{array}{l}\text { These transgenic mice express Cre } \\
\text { recombinase under the control of a mouse } \\
\text { Tek promoter and enhancer. This promoter } \\
\text { is active in endothelial cells. }\end{array}$ \\
\hline
\end{tabular}

\section{Reagents}

1. Sterile Phosphate-buffered saline (PBS, 1×, $\mathrm{pH} 7.2$ )

2. Ultra-purified water

3. Disinfectant: $70 \%$ ethanol solution ( $70 \mathrm{ml}$ of $100 \%$ ethanol added to $30 \mathrm{ml}$ of water)

\section{Equipment}

Note: All equipment may be substituted with appropriate alternatives from other manufacturers. 


\section{General Equipment}

1. Appropriate microscope stage (Zeiss LSM710 motorized $X, Y$ stage with Z focus) with heating pad (FHC Inc., catalog number: 40-90-2-07, Bowdoin, ME, USA)

2. Gas anesthesia vaporizer (IsoTec4; Datex-Ohmeda GE Healthcare, Waukesha, WI, USA)

3. Oxygen gas

4. Hair removal cream (Nair ${ }^{\circledR}$ hair remover cream for face)

5. Curved blunt forceps (VWR, catalog number 76319-850)

6. Fine forceps (VWR, catalog number 82027-408)

7. Scissors

ICaution: Only use the tip of sharp scissors when conducting fine neonatal mouse surgery. This will allow for more precise work with less tissue damage.

8. Solder lug terminal; $0.3 \mathrm{~mm}$; M4 (Manufacturer OSTERRATH, manufacturer part number 602814-51/0030, Figure2A)

9. Holding devices for both the cover glass and solder lug terminal (TEKTON 7521 Helping Hand with Magnifier, Figure 2C)

\section{Microscope}

1. Inverted two-photon laser scanning microscope (TPLSM, e.g., Zeiss LSM710)

2. Laser: Ti:Sapphire Chameleon Vision (Coherent)

3. Objectives: 20× (Water immersion lens, e.g., Zeiss W Plan APOCHROMAT, 1.0 DIC (UV) VIS$\mathrm{IR} \infty / 0.17)$

4. Software application: ZEN (Zeiss, imaging software)

Note: Magnifiers are not needed in this study and can be removed from the device.

\section{Anesthesia}

1. Portable anesthesia machine

2. Isoflurane vaporizer

3. Anesthesia breathing circuit

4. $\mathrm{O}_{2}$ gas flow regulator for E-cylinders (Praxair)

5. $\mathrm{O}_{2}$ tank (E-cylinder)

6. Anesthesia breathing circuit and nose cone

\section{Procedure}

\section{Stage setup}

1. Make sure the heating pad of the stage is plugged in and that the stage is at $37^{\circ} \mathrm{C}$ before starting the animal procedure. 


\section{Microscope setup}

2. Turn on the laser source, microscope and start the imaging applications, and all imaging components (data analysis workstation/computer, monitor, laser sources, camera, raster scanning unit, and detectors).

ICAUTION: Prepare all microscopic settings before subjecting the animals to anesthesia.

\section{Anesthetic cone setup}

3. Uncouple the normal sized anesthetic cone from the anesthetic breathing circuit and cover the end with a cut-out finger from a rubber glove. Cut a small hole at the tip of the rubber finger to fit the size of the neonatal mouse head (Figure 1A).

ICAUTION: The hole size of the rubber glove finger should fit tightly around the head of the neonate mouse head, otherwise the isoflurane gas might leak.

\section{Mice and surgery for image preparation}

4. A neonatal $R O S A^{m T / m G}$;Tie2-Cre mouse of either sex is used in this protocol to visualize the hemodynamics of the small intestinal microcirculation.

5. Place the mouse in the chamber for anesthesia with $2 \%$ isoflurane in oxygen. Confirm appropriate anesthesia by the absence of the withdrawal reflex after a toe pinch, as well as physiological responses including reduced respiratory and heartbeat rate. Once the mouse is anesthetized, reduce the isoflurane gas flow to $1-1.5 \%$.

6. Put the mouse on the preheated heating pad on a diaper pad, and the mouse head into the end of the anesthetic breathing circuit, with the mouse's upper extremities fixed to the rubber finger with tape (Figure 1B).

ICAUTION: To ensure that the animal is unconscious during the procedure, isoflurane is delivered continuously via the end of the anesthetic breathing circuit with the rubber finger covering the nose.

7. Tape the lower extremities of the mouse to fix the body on the stage of the microscope and position it with a downward slope towards the tail (Figure 1C).

8. The level of the downward slope should be such that it makes a triangular space on the neonatal abdomen from a lateral view. The angle $\theta$ from 30 to 45 degree is the best range for this experiment (Figure 1D, see also Table 2).

$\triangle$ CRITICAL STEP: Making this triangular space is critical to stabilize the image or movie. The top side of this triangle should be aligned at the same height as the sternum, and the bottom of the angle should be positioned above the Anterior Superior lliac Spine (Figure 1D). 
Table 2. Troubleshooting table

\begin{tabular}{llll}
\hline Step & Problem & Possible reason & Solution \\
\hline $\mathbf{8}$ & $\begin{array}{l}\text { Can not make the triangle } \\
\text { space }\end{array}$ & $\begin{array}{l}\text { The level of downward slope } \\
\text { is not enough }\end{array}$ & $\begin{array}{l}\text { Put a small back pillow made by Kimwipe or } \\
\text { a small folded cloth between the soft } \\
\text { absorbable pad and mouse's back. See also } \\
\text { Figure 1D. }\end{array}$ \\
\hline & $\begin{array}{l}\text { Bleeding from the abdominal } \\
\text { wall }\end{array}$ & $\begin{array}{l}\text { Incision line make a muscle incision vertically at the } \\
\text { transversed the inferior } \\
\text { epigastric arteryand vein }\end{array}$ & $\begin{array}{l}\text { middle of abdominal wall. You can distinguish } \\
\text { the vertical abdominal midline from other } \\
\text { muscle lesion because only the midline can } \\
\text { see through to the inside of the abdominal } \\
\text { wall clearly. }\end{array}$ \\
\hline
\end{tabular}

During picking up of the intestine and putting

13

Bleeding from the intestine or mesenteric lesion
Too much force was used to pulled the intestine outside of the abdominal cavity

it back into the abdominal cavity, be careful not to pull the intestine lower than the superior interior iliac spine level to avoid the intestinal and mesentric injury induced by tensile force.

\begin{tabular}{lll}
\hline Having a difficulty making the & $\begin{array}{l}\text { Fine manual work could be } \\
\text { difficult by hand }\end{array}$ \\
\hline
\end{tabular}

The fixed intestinal color is getting dark after putting the cover glass on it
The pressure on the fixed intestine is too high

Use two fine pliers and gently make the curve. Remove the cover glass quickly and release the compressed intestine. Re-check the diameter of both the intestine and the space created by the curved solder lug, and regulate the shape of the solder lug to fit the intestinal diameter.

Can not keep the optimal intestinal observation position without intestinal physical injury.
Pulling up the intestine over the heart level or pulling down the intestine over the superior anterior iliac spine level.

Re-check the anesthetic mouse body condition. Make sure that there is a enough trianglular square under the horizontal line of sternum. See also step 5 troubleshooting.

Too high of a laser power can cause not only photobleaching or photo-damage to the observed intestine but also non-specific autofluorescence. Decreasing the laser power (regulation of both laser intensity and fluorescent gain) is necessary, and then keep the imaging lesion clear as long as possible when long observation time is needed.

Control the $x$ and $y$ axes and find the Focusing area is outside of appropriate contact region. Possible intestinal Difficulty focusing the images the contact area coverglass. See also step 20.

9. Disinfect the whole abdominal skin area with $70 \%$ ethanol. Note that normally, mice have no hair in the neonatal period. If the mice have the hair on the operational skin area, you should use the hair remover gel to remove the hair softly with a small cotton swab. 
10. Put a sterile gauze on top of the disinfected abdominal area. The sterile gauze should have a small hole in the middle large enough to allow making a small incision.

11. Make a vertical skin incision at the lower to middle abdominal area. This incision will expose the intestine and urinary bladder through the abdominal muscle layer.

12. Cut the abdominal muscle and peritoneal layer vertically in the middle (Figure 1E, see also Table 2).

ICAUTION: The length of the abdominal muscle incision should be shorter than the length of the skin incision to prevent intestinal protrusion and allow the mouse's body to maintain its position throughout the imaging.

13. Push gently on the lateral sides of the abdominal wall to allow a portion of the intestine to be completely externalized from the abdominal cavity. Using the cecum as a landmark, locate the segment of the small intestine that is of interest to your study, and place the rest of the intestinal length back into the abdominal cavity (Figure 1F, see also Table 2).

$\triangle$ CRITICAL STEP: It is not recommended to use forceps to locate the cecum because even with gentle maneuvers, picking with forceps could easily injure the neonatal intestine. Additionally, it is possible that with an intestinal disease model, the microcirculation of the target area is already compromised, making the intestine and blood vessels even more delicate. With confirmation of the location of the cecum, which allows to distinguish the small intestine from the colon, the intestine should be carefully handled from a region away from the target area. The rest of the intestine should be put back into the abdominal cavity using blunt forceps.

ICAUTION: Gentle maneuvers are necessary while moving the intestine around. Using blunt forceps, try to move the intestine by grasping tissue away from target region. Avoid unnecessary picking of the intestine with the forceps to reduce the number of contact points and to prevent puncturing/injuring the intestine. Avoid grasping the mesentery when moving the intestine.

14. For the small intestine section that is selected for observation, use blunt forceps to gently pull out a small portion of the intestine that you will be observing. It is important to do this gently and allow the tissue to be under no tension to prevent potential ischemia or damage. Place one drop of PBS on the intestine to avoid drying. 
A

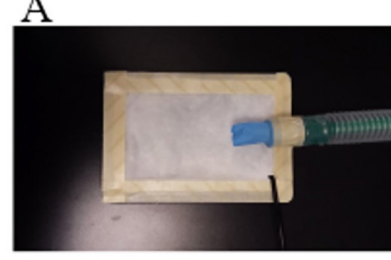

C

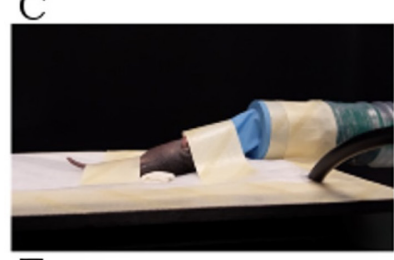

E

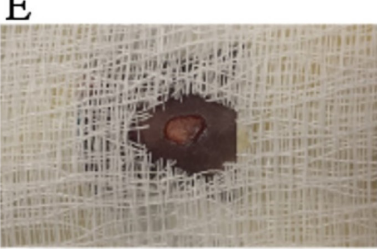

$\mathrm{B}$

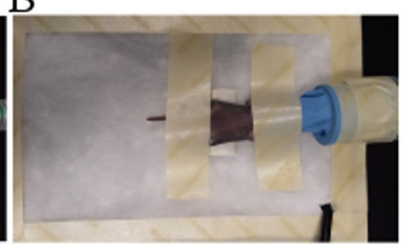

$\mathrm{D}$

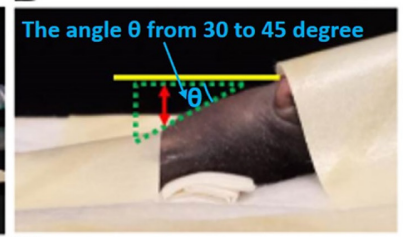

F

Figure 1. Neonatal mouse preparation for the TPLSM analysis. (A-D) Mouse anesthesia and appropriate positioning. (E, F) Intestine exposure.

\section{Preparation of custom fixing device}

15. Bend the solder lug to a gentle curve, creating a space to sandwich the intestine between the ring of the solder lug and the cover glass (Figures 2A, 2B).

16. Attach the other end of the bent solder lug to the holding device with tape (Figure 2C).

17. Put the cover glass on the end of the solder lug that is attached to the holding device and clip it in place using the holding device. Ensure that there is space between the ring of the solder lug and the cover glass. The thickness of the space between the cover glass and the ring of the solder lug should be exactly the same as the maximum diameter of the intestine (Figure 2D, see also Table 2).

ICAUTION: Maintaining a fixed space between the solder lug and cover glass according to the actual diameter of the intestine is crucial to keep the intestine in place while allowing proper intestinal blood circulation.

18. Bend the ring of the solder lug to a complete horizontal plane parallel to the cover glass (Figure $2 \mathrm{E}, 2 \mathrm{~F})$.

$\triangle$ CRITICAL STEP: Precise bending of the solder lug to sandwich the intestine between the ring of the solder lug and the cover glass is crucial to keep the small intestine in position during microscopic observation.

19. After creating the appropriate space between the bent solder lug and cover glass and orienting the solder lug ring parallel to the cover glass on a horizontal plane, remove the cover glass. 

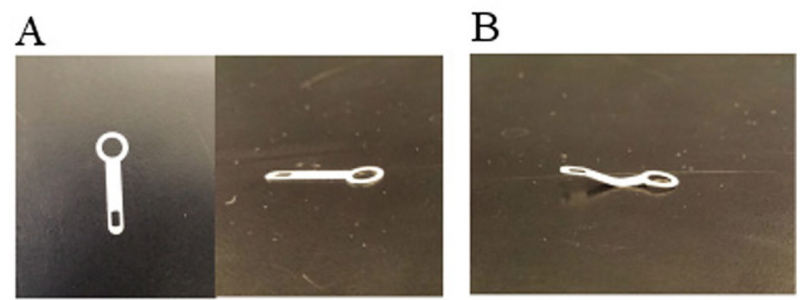

C

$\mathrm{D}$
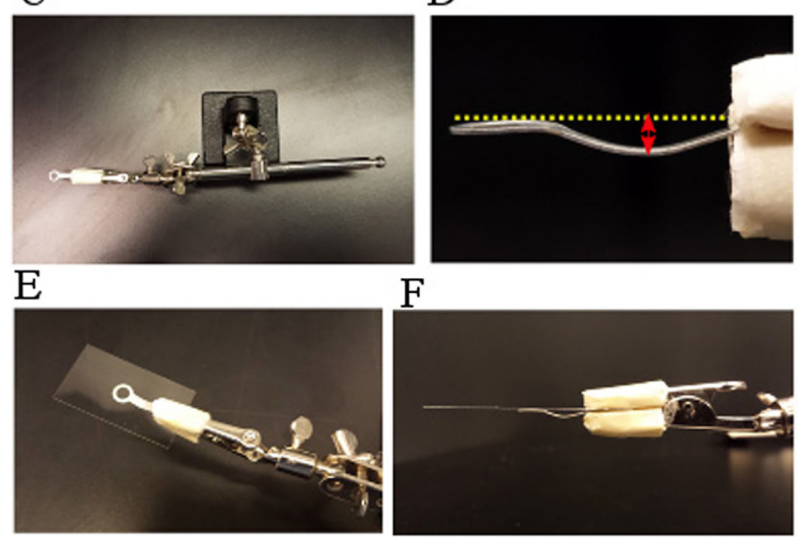

Figure 2. Fixing device setup for intestinal microscopic observation. (A, B) Solder lug. (C, D). Solder lug attached to holding device with appropriate spacing, the spacing should be the same as the diameter of the intestine being used (generally between 1 and $3 \mathrm{~mm}$ ). (E, F) Cover glass attached on top of the solder lug and clipped with the holding device.

\section{Preparation of the microscopic stage}

20. Transfer the customized fixing device to the microscopic stage where the mouse is mounted.

21. Put the ' $U$ ' shaped intestine on the ring of the solder lug. Wet the intestine with a drop of PBS and place the cover glass directly on top of the area, fixing the cover glass with the clip of the holding device. The bottom area of the ' $U$ ' shaped intestine should be slightly over the solder lug (Figure 3A).

$\triangle$ CRITICAL STEP: If the bottom area of ' $U$ ' shaped intestine is not over the solder lug, the position of the intestine should be moved, as miss positioning may prevent stable intravital imaging. 
A

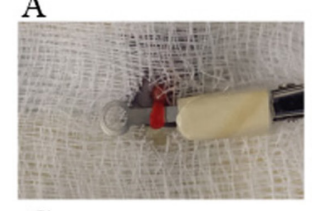

$\mathrm{C}$

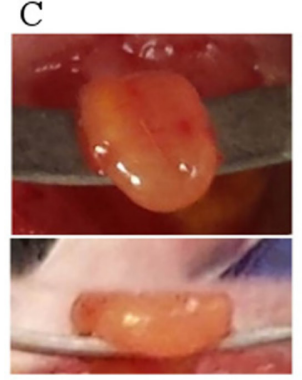

B
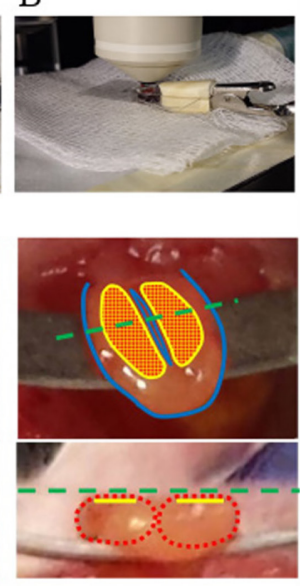

$\mathrm{D}$

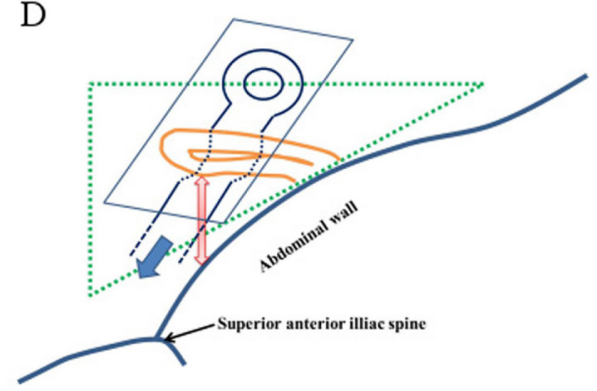

Figure 3. Neonatal mouse intestine exposed for TPLSM observation. (A-C) Mouse intestine preparation and areas of observation. The yellow marking or alternatively in the red dotted area shows the contact lesion area between small intestinal wall and the cover glass. The green dashed line (under image) shows the cover glass level (the top picture in C: view from above the cover glass, the bottom picture in $\mathrm{C}$ : view from the frontal view. The observable area is between the green dotted line and the yellow line from the frontal view. The red dashed circle represents the outer intestinal wall line from the frontal view. (D) Illustration of mouse intestine for microscopic observation.

22. Put one drop of distilled water on the cover glass where the intestine is touching the cover glass. Bring down the objective lens immersion into the waterdrop dome and begin the microscopic observation (Figure 3B).

23. Figure $3 \mathrm{C}$ shows the ideal set up of the customized holding device, the microscope, and the mouse. After putting the cover glass on top of the intestine, only a small portion of the intestine will be in contact with the cover glass (shown in Figure $3 \mathrm{C}$ inside the yellow marking or alternatively in the red dotted area). This area will be the best location to observe the whole intestinal wall from the serosa level to the villi. When viewing the set up from the side, the segment of intestine sandwiched between the solder lug and the cover glass should look slightly oval shaped (Figure $3 \mathrm{C}$ red dotted circles). This positioning of the intestine is essential to prevent ischemia (see also Table 2). 
$\triangle$ CRITICAL STEP: Make sure that the intestine is not damaged after fixation on the device and putting on the coverglass. Damaged tissue can be identified by increased redness. If the color of the intestine turns dark soon after fixation, remove the cover glass immediately and release the compression to restore intestinal blood flow circulation.

ICAUTION: Placing of the cover glass and securing it with the clip must be done very carefully. Applying too much pressure with the clip can break the cover glass.

24. Once the intestine is fixed, ensure that any parts of the device are not in direct contact with the mouse body (Figure 3D). Additionally, confirm that the fixed intestine that is being observed lies between the sternum and the Anterior Superior Iliac Spine in position (see also Table 2).

ICAUTION: Do not pull the intestine above the level of the heart (towards the head) to avoid both the potential positional effects on blood pressure and the potential effects on blood flow from stretching the tissue. As well, do not pull the intestine below the level of the Anterior Superior lliac Spine, this would impose too much tension and cause ischemia.

\section{Intravital imaging}

25. Intravital observation can now be performed using a Zeiss LPM710 inverted microscope (Zeiss) with $\times 20$ water immersion objective lens (W Plan-Apochromat 20×/1.0 DIC, VIS-IR M27 75mm). TPLSM images are acquired at $512 \times 512$ pixels of spatial resolution from a $386.45 \mu \mathrm{m}$ field of view. The excitation wavelength to detect GFP is $910 \mathrm{~nm}$. The scan speed should be set at 1.27 $\mu s /$ pixel. Two-photon fluorescence signals are collected by an internal detector (non-descanned detection method) at an excitation wavelength.

ICAUTION: The laser power is adjusted according to the imaging depth and intestinal diameter. When imaging at greater depths, the laser power level should be increased (up to 100\%) manually using the laser power level controller. The laser power should be adjusted slowly from a low to high level to avoid photo-bleaching of the target area, which may occur at higher laser power. If the observation area is photo-bleached the stage must be moved to focus on another area.

26. Each area of interest is scanned at a high magnification (water-immersion objective $20 \times$ or higher if desired) by manually setting the $X / Y$ plane and adjusting the $Z$ axis to obtain highresolution, clear TPLSM images.

27. In our experimental setting, the imaging depth ranged from $100 \mu \mathrm{m}$ to $400 \mu \mathrm{m}$. Optimal highresolution images were obtained from the tissue surface up to $200 \mu \mathrm{m}$ in depth. For optimal simultaneous imaging of EGFP and tdTOMATO, detection gain should be adjusted for EGFP (to 500-570) or tdTOMATO (to 580-650)

ICAUTION: The range of gain value should be changed according to each intestinal region. A higher gain produces a stronger signal; however, it also produces more background.

$\triangle$ CRITICAL STEP: The combination of laser power and gain value determines the intensity of the laser signal. The settings of these two values depend on the quality of image focus and on the length of time of continuous imaging (see also Table 2). 
28. Start the observation and recording by TPLSM and save the acquired data. Saving the data as a czi file is recommended because czi files can be used to analyze the blood flow dynamics or make Z-stack movies/3-dimentional images using ZEN 2 lite software after imaging.

ICAUTION: For the analysis of blood flow dynamics, including blood flow velocity, blood flow volume, and leukocyte rolling speed, we recommend capturing 80-100 frames within $30 \mathrm{~s}$.

29. After microscopic intravital observations, euthanize the mouse under general anesthesia according to approved protocols.

\section{Data analysis}

\section{Processing of image data}

1. Launch the ZEN 2.0 lite software (freely available from the ZEISS company website after registration).

2. Open the saved czi files and place a properly sized scale bar. The czi file contains information about each imaging setting, as well as scanned area size and time. To create image data from sequential time course images, choose one image and select the 'export/import' button from the file tag. Then, select 'export' and choose the appropriate file type (e.g., JPEG, TIFF, PNG etc.). To create a movie from the data, select the 'movie export' function and choose the appropriate file type (e.g., AVI, WMF, MOV etc.). The czi files can be used for the analysis.

\section{Blood flow velocity}

The blood flow velocity $(\mathrm{V})$ is calculated as described in several studies that quantified the blood flow in vessels of different caliber, from arteries to capillaries (Tang et al., 2015). In one movie (20$30 \mathrm{~s}$ ), select a sequence of the four to six most clear images of a blood vessel. Measure the tangent length that is parallel to the blood flow direction $(\Delta X)$ and the cosine length of the strand that is vertical to the blood flow direction $(\Delta T)$. The blood flow velocity is calculated using the following formula (Figures 4A, 4B and Figure 5):

$V[\mu m / m s]=\Delta X / \Delta T$

Velocity can be defined as a mean value calculated from four or six strands selected from the movie. 


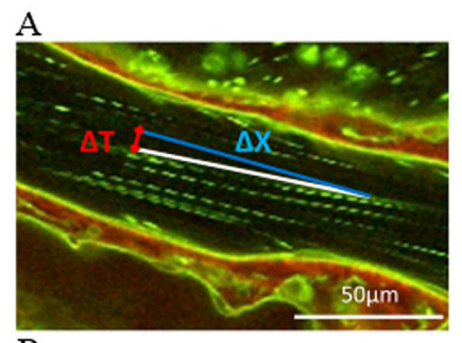

B
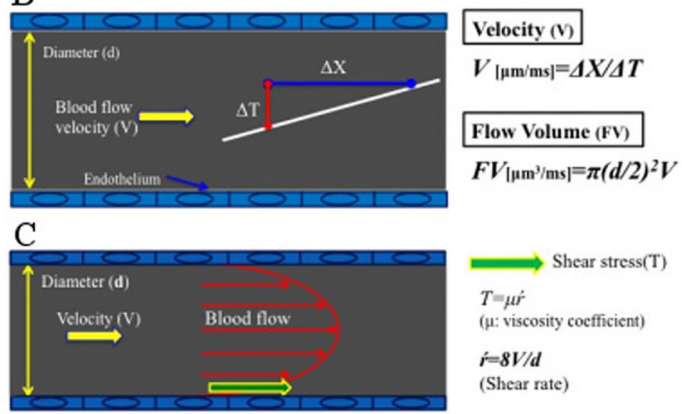

$T=\mu \dot{r}$
$(\mu$ : viscosity coefficient)

$r=8 V / d$

(Shear rate)

$\mathrm{D}$
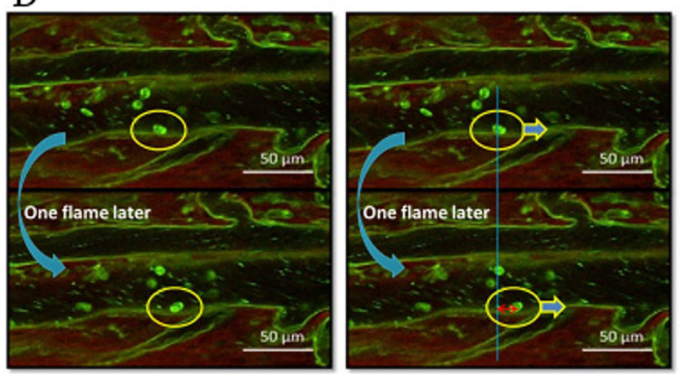

Figure 4. Dynamic intestinal microcirculation in neonatal mice. A. Intestinal microcirculation image obtained by TPLSM. B. Blood flow volume calculation. C. Blood vessel wall shear rate calculation. D. Leukocyte rolling speed calculation.

\section{Blood flow volume}

Blood flow volume $(F V)$ is calculated using the following formula (Figure $4 \mathrm{~B}$ and Figure 5):

$F V\left[\mu m^{3} / m s\right]=\pi(d / 2)^{2} \Delta X / \Delta T$

[d] is the maximum diameter of the blood vessel

\section{Shear rate of blood vessel wall}

Blood vessel wall shear rate (W) is calculated in a 100- $\mu \mathrm{m}$ segment of the vessel using the formula based on the Newtonian definition (Russell et al., 2003) (Figure 4C and Figure 5):

$W[1 / m s]=8 \mathrm{~V} / \mathrm{d}$ 

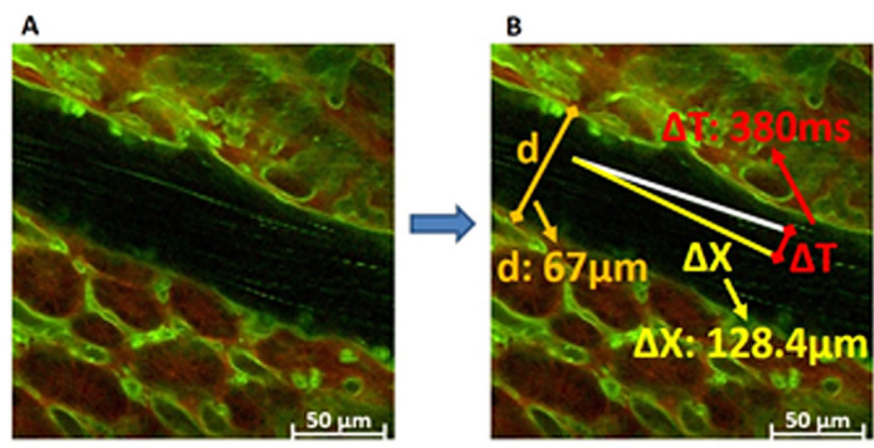

C

Velocity $(V)=\Delta X / \Delta T=128.4 / 380=3.38 \times 10^{-1}[\mu \mathrm{m} / \mathrm{ms}]$

Flow volume $(\mathrm{FV})=\pi(\mathrm{d} / 2)^{2} \Delta X / \Delta T=\pi(67 / 2)^{2} \times 3.38 \times 10^{-1}=1.19 \times 10^{3}\left[\mu \mathrm{m}^{3} / \mathrm{ms}\right]$

Shear rate $(\mathrm{W})=8 \mathrm{~V} / \mathrm{d}=8 \times 3.38 \times 10^{-1} / 67=4.04 \times 10^{-2}[1 / \mathrm{ms}]$

Figure 5. Example of experimental result and data analysis. A. One still/flame image cutting from the recorded video (one flame scan speed: $380 \mathrm{~ms}$ ). From this find a straight line of the platelet. B. Measure the tangent length of the straight line that is parallel to the blood flow direction $(\Delta \mathrm{X})$, scan time $(\Delta \mathrm{T})$, and blood vessel maximum diameter (d). C. Calculate the velocity, flow volume, and shear rate using the specific formula.

\section{Number of adherent leukocytes}

Adherent leukocytes can be defined in each vessel segment as cells that do not move or detach from the endothelial lining within a specified observation period of 5-10 s. Quantification is done by counting the number of adherent leukocytes sticking to the endothelial surface within a $100-\mu \mathrm{m}$ length of a single vessel (Kubes et al., 2003; Nakagawa et al., 2006).

\section{Leukocytes rolling speed}

Leukocytes' rolling phenomenon is observed mainly in the post capillary venule. The post capillary venule is a V1 or V2 level vein that is located in the submucosal level, made from the combined branches of the capillary vessels (Yu et al., 2009). The maximum diameter of this venule in the neonatal mouse intestine is approximately $20-60 \mu \mathrm{m}$. Choose a frame in which there is a consistent maximum diameter of post-capillary venules and select a single rolling leukocyte to follow as it rolls on the inner venule wall. Measure the distance travelled by the leukocyte and record the length of travel time, as determined by the time from the first frame observed to the last frame observed. Leukocyte rolling speed $[\mu \mathrm{m} / \mathrm{ms}]$ is calculated in $[\mu \mathrm{m} / \mathrm{ms}]$ (Figure $4 \mathrm{D}$ ):

Leukocyte rolling speed $=[\mu \mathrm{m} / \mathrm{ms}]$

Where: leukocyte traveled distance length $[\mu \mathrm{m}] /$ the scan time span between the two selected time frames [ms]. 
Please cite this article as: Koike et. al., (2021). Live Intravital Intestine with Blood Flow Visualization in Neonatal Mice Using Two-photon Laser Scanning

\section{Anticipated results}

By using this method of TPLSM imaging of the neonatal intestine, we are able to observe and analyze the microcirculation and blood flow dynamics of the neonatal small intestine in detail (Video 1). Video 1 starts at tips of the villi and travels downwards towards the based of the villi and then through the underlying vascular supply and smooth musculature at the end. Moreover, acquisition of z-stack and $x-y$ data allows creating three-dimensional images of the entire structure of the small intestine, from the top of the villi to the bottom, including the submucosal area. Administration of additional fluorescent dyes such as SYTOX green or red prior to imaging enables the localization of affected cells in a disease model (Video 2). Using this approach, we revealed that the top of the villi is the main area affected by necrosis in the experimental NEC mouse model (Video 2). This approach is also useful for long-term analysis of the effects of drugs and other procedures on the neonatal small intestine. Furthermore, this approach allows the user to visualize changes in intestinal microcirculation in a target area.

In summary, the combination of the organ stabilizing method for TPLSM described in this protocol yields reliable results for studying neonatal intestine development and intestinal pathophysiology.

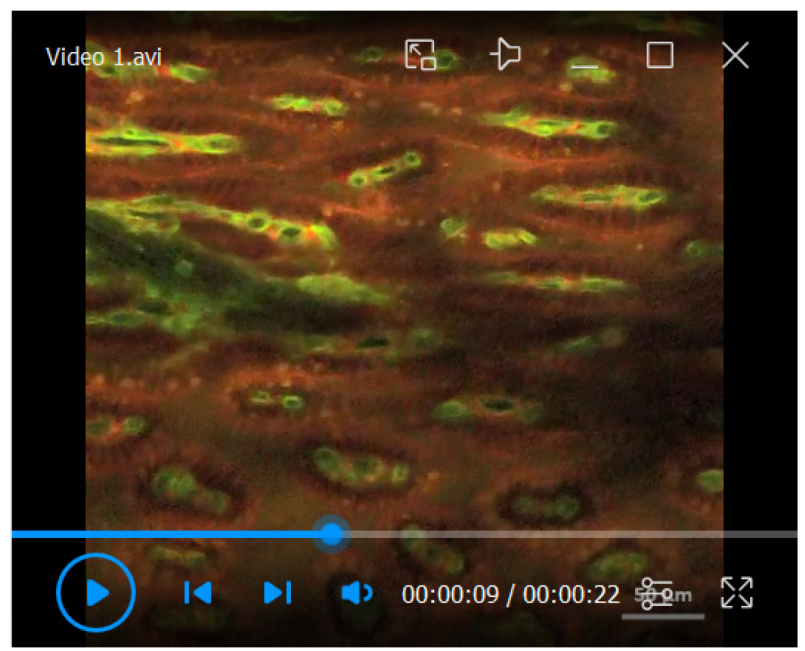

Video 1. Microcirculation of the neonatal small intestine 


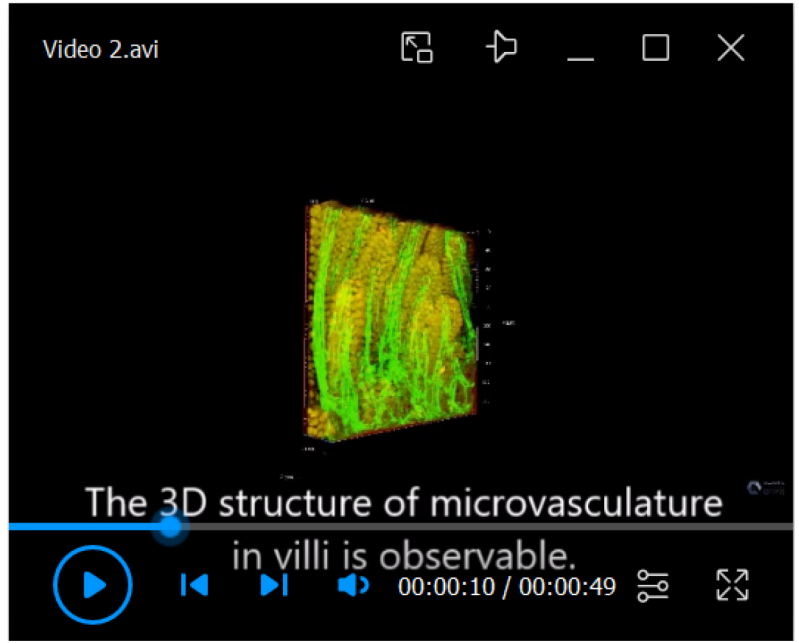

Video 2. 3-D image of the whole villi in a control neonatal mouse and the necrotizing enterocolitis mouse model

\section{Acknowledgments}

BL is the recipient of Restracomp Fellowship, The Hospital for Sick Children and Early Career Award Program grant from Thrasher Research Fund (14503). PDO is supported by the Canadian Institutes of Health Research (CIHR) (162208 and 149046), the Heart and Stroke Foundation of Canada (G17- 0018613), and the Natural Sciences and Engineering Research Council of Canada (NSERC) (500865). AP is the recipient of a Canadian Institutes of Health Research (CIHR) Foundation Grant 353857.

Author contributions: Y.K., B.L., Y.C., N.G., M.A., H.M., C.L., A.H., and R.W.: Conception and design, Collection and/or assembly of data, Data analysis and interpretation, Manuscript writing, Final approval of manuscript. K.U., M.I., and P.D.O. Conception and design, Final approval of manuscript. A. P.: Conception and design, Financial support, Final approval of manuscript.

\section{Competing interests}

The authors have no conflict of interest to declare.

\section{Ethics}

All procedures described in this protocol were approved by the Institutional Animal Care Committee at the Toronto Medical Discovery Tower (No.4886.0), and by the Advanced Optical Microscopy Facility. 
Please cite this article as: Koike et. al., (2021). Live Intravital Intestine with Blood Flow Visualization in Neonatal Mice Using Two-photon Laser Scanning

\section{References}

1. Barlow, B., Santulli, T. V., Heird, W. C., Pitt, J., Blanc, W. A. and Schullinger, J. N. (1974). An experimental study of acute neonatal enterocolitis--the importance of breast milk. $J$ Pediatr Surg 9(5): 587-595.

2. Cao, L., Kobayakawa, S., Yoshiki, A. and Abe, K. (2012). High resolution intravital imaging of subcellular structures of mouse abdominal organs using a microstage device. PLoS One 7(3): e33876.

3. Coppieters, K., Martinic, M. M., Kiosses, W. B., Amirian, N. and von Herrath, M. (2010). A novel technique for the in vivo imaging of autoimmune diabetes development in the pancreas by twophoton microscopy. PLoS One 5(12): e15732.

4. Devi, S., Li, A., Westhorpe, C. L., Lo, C. Y., Abeynaike, L. D., Snelgrove, S. L., Hall, P., Ooi, J. D., Sobey, C. G., Kitching, A. R. and Hickey, M. J. (2013). Multiphoton imaging reveals a new leukocyte recruitment paradigm in the glomerulus. Nat Med 19(1): 107-112.

5. Ferrer, M., Martin-Jaular, L., Calvo, M. and del Portillo, H. A. (2012). Intravital microscopy of the spleen: quantitative analysis of parasite mobility and blood flow. $J$ Vis $\operatorname{Exp}(59)$.

6. Hackl, M. J., Burford, J. L., Villanueva, K., Lam, L., Susztak, K., Schermer, B., Benzing, T. and Peti-Peterdi, J. (2013). Tracking the fate of glomerular epithelial cells in vivo using serial multiphoton imaging in new mouse models with fluorescent lineage tags. Nat Med 19(12): 16611666.

7. Honda, M., Takeichi, T., Asonuma, K., Tanaka, K., Kusunoki, M. and Inomata, Y. (2013). Intravital imaging of neutrophil recruitment in hepatic ischemia-reperfusion injury in mice. Transplantation 95(4): 551-558.

8. Koike, Y., Tanaka, K., Okugawa, Y., Morimoto, Y., Toiyama, Y., Uchida, K., Miki, C., Mizoguchi, A. and Kusunoki, M. (2011). In vivo real-time two-photon microscopic imaging of platelet aggregation induced by selective laser irradiation to the endothelium created in the beta-actingreen fluorescent protein transgenic mice. J Thromb Thrombolysis 32(2): 138-145.

9. Koike, Y., Uchida, K., Tanaka, K., Ide, S., Otake, K., Okita, Y., Inoue, M., Araki, T., Mizoguchi, A. and Kusunoki, M. (2014). Dynamic pathology for circulating free DNA in a dextran sodium sulfate colitis mouse model. Pediatr Surg Int 30(12): 1199-1206.

10. Kubes, P., Heit, B., van Marle, G., Johnston, J. B., Knight, D., Khan, A. and Power, C. (2003). In vivo impairment of neutrophil recruitment during lentivirus infection. J Immunol 171(9): 48014808.

11. Liang, X., Grice, J. E., Zhu, Y., Liu, D., Sanchez, W. Y., Li, Z., Crawford, D. H., Le Couteur, D. G., Cogger, V. C., Liu, X., Xu, Z. P. and Roberts, M. S. (2015). Intravital multiphoton imaging of the selective uptake of water-dispersible quantum dots into sinusoidal liver cells. Small 11(14): 1711-1720.

12. Looney, M. R., Thornton, E. E., Sen, D., Lamm, W. J., Glenny, R. W. and Krummel, M. F. (2011). Stabilized imaging of immune surveillance in the mouse lung. Nat Methods 8(1): 91-96. 
Please cite this article as: Koike et. al., (2021). Live Intravital Intestine with Blood Flow Visualization in Neonatal Mice Using Two-photon Laser Scanning

13. Lu, H. H., Wu, Y. M., Chang, W. T., Luo, T., Yang, Y. C., Cho, H. D. and Liau, I. (2014). Molecular imaging of ischemia and reperfusion in vivo with mitochondrial autofluorescence. Anal Chem 86(10): 5024-5031.

14. Martinic, M. M. and von Herrath, M. G. (2008). Real-time imaging of the pancreas during development of diabetes. Immunol Rev 221: 200-213.

15. McDole, J. R., Wheeler, L. W., McDonald, K. G., Wang, B., Konjufca, V., Knoop, K. A., Newberry, R. D. and Miller, M. J. (2012). Goblet cells deliver luminal antigen to $C D 103^{+}$dendritic cells in the small intestine. Nature 483(7389): 345-349.

16. Morgan, J., Young, L. and McGuire, W. (2014). Delayed introduction of progressive enteral feeds to prevent necrotising enterocolitis in very low birth weight infants. Cochrane Database Syst Rev(12): CD001970.

17. Nakagawa, N. K., Nogueira, R. A., Correia, C. J., Shiwa, S. R., Costa Cruz, J. W., Poli de Figueiredo, L. F., Rocha, E. S. M. and Sannomiya, P. (2006). Leukocyte-endothelium interactions after hemorrhagic shock/reperfusion and cecal ligation/puncture: an intravital microscopic study in rat mesentery. Shock 26(2): 180-186.

18. Peti-Peterdi, J., Burford, J. L. and Hackl, M. J. (2012). The first decade of using multiphoton microscopy for high-power kidney imaging. Am J Physiol Renal Physiol 302(2): F227-233.

19. Pezzati, M., Dani, C., Tronchin, M., Filippi, L., Rossi, S. and Rubaltelli, F. F. (2004). Prediction of early tolerance to enteral feeding by measurement of superior mesenteric artery blood flow velocity: appropriate- versus small-for-gestational-age preterm infants. Acta Paediatr 93(6): 797-802.

20. Pittet, M. J. and Weissleder, R. (2011). Intravital imaging. Cell 147(5): 983-991.

21. Ritsma, L., Steller, E. J., Ellenbroek, S. I., Kranenburg, O., Borel Rinkes, I. H. and van Rheenen, J. (2013). Surgical implantation of an abdominal imaging window for intravital microscopy. Nat Protoc 8(3): 583-594.

22. Russell, J., Cooper, D., Tailor, A., Stokes, K. Y. and Granger, D. N. (2003). Low venular shear rates promote leukocyte-dependent recruitment of adherent platelets. Am J Physiol Gastrointest Liver Physiol 284(1): G123-129.

23. SchiessI, I. M. and Castrop, H. (2013). Angiotensin II AT2 receptor activation attenuates AT1 receptor-induced increases in the glomerular filtration of albumin: a multiphoton microscopy study. Am J Physiol Renal Physiol 305(8): F1189-1200.

24. Stappenbeck, T. S., Hooper, L. V. and Gordon, J. I. (2002). Developmental regulation of intestinal angiogenesis by indigenous microbes via Paneth cells. Proc Natl Acad Sci U S A 99(24): 15451-15455.

25. Tanaka, K., Koike, Y., Shimura, T., Okigami, M., Ide, S., Toiyama, Y., Okugawa, Y., Inoue, Y., Araki, T., Uchida, K., Mohri, Y., Mizoguchi, A. and Kusunoki, M. (2014a). In vivo characterization of neutrophil extracellular traps in various organs of a murine sepsis model. PLoS One 9(11): e111888. 
Please cite this article as: Koike et. al., (2021). Live Intravital Intestine with Blood Flow Visualization in Neonatal Mice Using Two-photon Laser Scanning

26. Tanaka, K., Toiyama, Y., Okugawa, Y., Okigami, M., Inoue, Y., Uchida, K., Araki, T., Mohri, Y., Mizoguchi, A. and Kusunoki, M. (2014b). In vivo optical imaging of cancer metastasis using multiphoton microscopy: a short review. Am J Transl Res 6(3): 179-187.

27. Tang, P., Zhang, Y., Chen, C., Ji, X., Ju, F., Liu, X., Gan, W. B., He, Z., Zhang, S., Li, W. and Zhang, L. (2015). In vivo two-photon imaging of axonal dieback, blood flow, and calcium influx with methylprednisolone therapy after spinal cord injury. Sci Rep 5: 9691.

28. Thompson, A., Silva, C. T., Gork, A. S., Wang, D. and Ehrenkranz, R. A. (2014). Intestinal blood flow by Doppler ultrasound: the impact of gestational age and time from first enteral feeding in preterm neonates. Am J Perinatol 31(4): 261-268.

29. Toiyama, Y., Mizoguchi, A., Okugawa, Y., Koike, Y., Morimoto, Y., Araki, T., Uchida, K., Tanaka, K., Nakashima, H., Hibi, M., Kimura, K., Inoue, Y., Miki, C. and Kusunoki, M. (2010). Intravital imaging of DSS-induced cecal mucosal damage in GFP-transgenic mice using two-photon microscopy. J Gastroenterol 45(5): 544-553.

30. Watkins, D. J. and Besner, G. E. (2013). The role of the intestinal microcirculation in necrotizing enterocolitis. Semin Pediatr Surg 22(2): 83-87.

31. Watson, A. J., Chu, S., Sieck, L., Gerasimenko, O., Bullen, T., Campbell, F., McKenna, M., Rose, T. and Montrose, M. H. (2005). Epithelial barrier function in vivo is sustained despite gaps in epithelial layers. Gastroenterology 129(3): 902-912.

32. Yazji, I., Sodhi, C. P., Lee, E. K., Good, M., Egan, C. E., Afrazi, A., Neal, M. D., Jia, H., Lin, J., Ma, C., Branca, M. F., Prindle, T., Richardson, W. M., Ozolek, J., Billiar, T. R., Binion, D. G., Gladwin, M. T. and Hackam, D. J. (2013). Endothelial TLR4 activation impairs intestinal microcirculatory perfusion in necrotizing enterocolitis via eNOS-NO-nitrite signaling. Proc Natl Acad Sci U S A 110(23): 9451-9456.

33. Yu, X., Radulescu, A., Zorko, N. and Besner, G. E. (2009). Heparin-binding EGF-like growth factor increases intestinal microvascular blood flow in necrotizing enterocolitis. Gastroenterology 137(1): 221-230.

34. Zani, A., Cordischi, L., Cananzi, M., De Coppi, P., Smith, V. V., Eaton, S. and Pierro, A. (2008). Assessment of a neonatal rat model of necrotizing enterocolitis. Eur J Pediatr Surg 18(6): 423426. 